\title{
Shape Designing of Engineering Images Using Rational Spline Interpolation
}

\author{
Muhammad Sarfraz, ${ }^{1}$ Munaza Ishaq, ${ }^{2}$ and Malik Zawwar Hussain ${ }^{2}$ \\ ${ }^{1}$ Department of Information Science, College of Computing Sciences and Engineering, Kuwait University, Adailiya Campus, Kuwait \\ ${ }^{2}$ Department of Mathematics, University of the Punjab, Lahore, Pakistan \\ Correspondence should be addressed to Muhammad Sarfraz; prof.m.sarfraz@gmail.com
}

Received 11 January 2015; Revised 17 February 2015; Accepted 17 February 2015

Academic Editor: Luigi Nicolais

Copyright @ 2015 Muhammad Sarfraz et al. This is an open access article distributed under the Creative Commons Attribution License, which permits unrestricted use, distribution, and reproduction in any medium, provided the original work is properly cited.

In modern days, engineers encounter a remarkable range of different engineering problems like study of structure, structure properties, and designing of different engineering images, for example, automotive images, aerospace industrial images, architectural designs, shipbuilding, and so forth. This paper purposes an interactive curve scheme for designing engineering images. The purposed scheme furnishes object designing not just in the area of engineering, but it is equally useful for other areas including image processing (IP), Computer Graphics (CG), Computer-Aided Engineering (CAE), Computer-Aided Manufacturing (CAM), and Computer-Aided Design (CAD). As a method, a piecewise rational cubic spline interpolant, with four shape parameters, has been purposed. The method provides effective results together with the effects of derivatives and shape parameters on the shape of the curves in a local and global manner. The spline method, due to its most generalized description, recovers various existing rational spline methods and serves as an alternative to various other methods including $v$-splines, gamma splines, weighted splines, and beta splines.

\section{Introduction}

Shape designing and reengineering plays a significant role in the area of Computer-Aided Engineering (CAE), Computer Graphics (CG), Computer-Aided Manufacturing (CAM), and Computer-Aided Design (CAD). It is also important for construction and reconstruction of several objects and also in the description of visual arts, medical, geological, physical, geographical, transportation, and various other phenomena. In particular, shape designing and reengineering of engineering images (see Figure 1) [1,2] makes an important contribution to material sciences and engineering studies. In addition, some other fields, including IP, CG, CAE, CAM, and CAD, make significant contribution. Designing of engineering images can also be related to another area called designing of objects using curves and surfaces which has its application in font design, computer animation, computational geometry, industrial art, industrial and architectural design, aerospace industries, and shipbuilding. A number of authors, in the current literature [1-27], have discussed numerous kinds of methods for curve and surface designing.

Designing and modeling of some appropriate curve scheme is one of the important phases of shape designing and reengineering of engineering images. The representation of planar objects, in terms of curves, has many advantages. For example, scaling, shearing, translation, rotation, and clipping operations can be performed without any difficulty. Although some amount of work has been done in the area, it is still desired to proceed further to explore more advanced and interactive strategies.

This paper is devoted for the development of computational method and mathematical modeling in engineering and material sciences, engineering material and design, mathematical physics, economics, optimization, and control. Engineering community can successfully apply the purposed method for optimal shape designing in 2 and 3 dimensions.

Sarfraz et al. [20] developed a rational cubic spline interpolant and used its scalar form to visualize the shape preserving $2 \mathrm{D}$ data. In this paper the parametric form of 

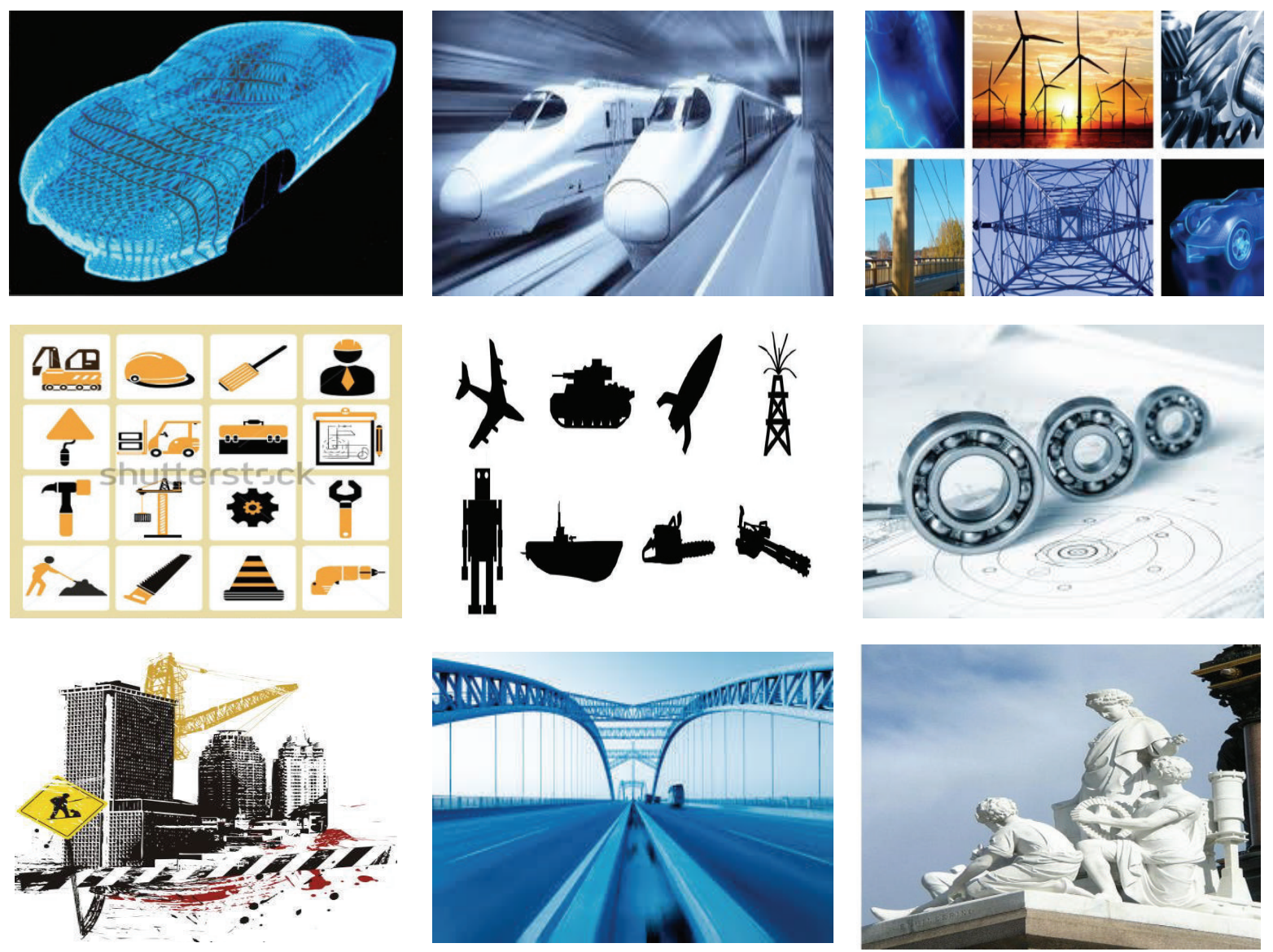

FIGURE 1: Some of the engineering images.

the rational cubic spline interpolant [20] is used for the designing of $2 \mathrm{D}$ images. The parameters in the description of interpolant allow freedom to the user and the designer for refining of the shape of objects. Practical examples are used to show that rational cubic spline interpolant is an effective designing scheme. Geometrical demonstrations of the properties like convex hull, variation diminishing, and interval and global tension properties are discoursed for rational cubic spline interpolant.

The proposed curve design scheme is distinct from the existing spline methods in various ways. It represents a parametric rational cubic spline with the most general description. This general description is quite helpful to produce well-controlled shape effects as compared to NURBS. This method not only provides a large variety of very interesting shape controls like biased, point, and interval tensions but, as special cases, also recovers various splines including the cubic spline curve [21], the rational cubic spline with tension [26], the rational cubic spline $[5,10,19]$. This method is also a stronger $C^{1}$ alternative to the $G C^{1}$ or $C^{1}$ spline methods like $v$-splines [23], beta splines [25], gamma splines [24], and weighted splines [22]. In this work, a constructive approach has been adopted to build a Hermite Bézier form for rational cubic spline curves with a simple $C^{1}$ continuity across the segments. The design curve possesses all the ideal geometric properties like partition of unity, convex hull, and variation diminishing. The method for evaluating this rational cubic spline curve is suggested by a transformation to BernsteinBézier form.

The paper is organized as follows. Section 2 discusses the preprocessing steps which include finding the boundary of planar objects and detection of corner points. Section 3 discusses the rational cubic spline method, its continuity, and geometrical shape properties with practical demonstrations. This section also includes continuity and geometrical shape properties of rational cubic spline. Description of derivative parameters is made in Section 4. Finally, Section 7 concludes the paper.

\section{Preprocessing Phases}

The engineering images can be designed either from scratch by directly working on the data points or by reengineering the already existing generic shapes to vector form. In both cases, a curve design scheme is required to apply. However, in the latter case, the proposed scheme starts with finding the boundary of the generic shapes and then using the output to find the corner points [7-9]. The image of the generic shapes can be acquired either by scanning or by some other means. The aim of boundary detection is to produce an object's shape in graphical or nonscalar representation. Demonstration of 


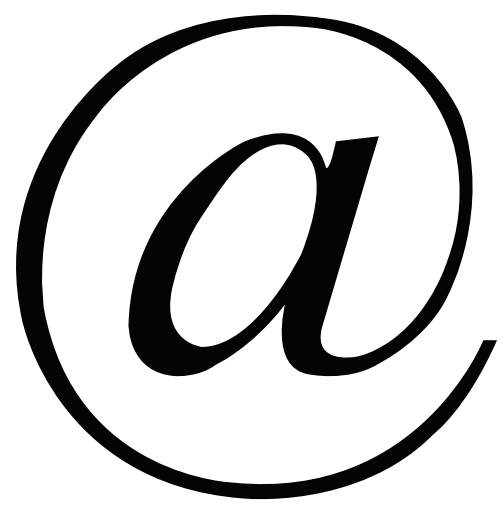

(a)

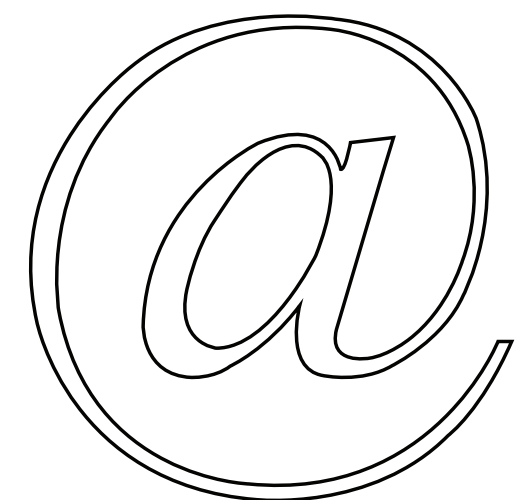

(b)

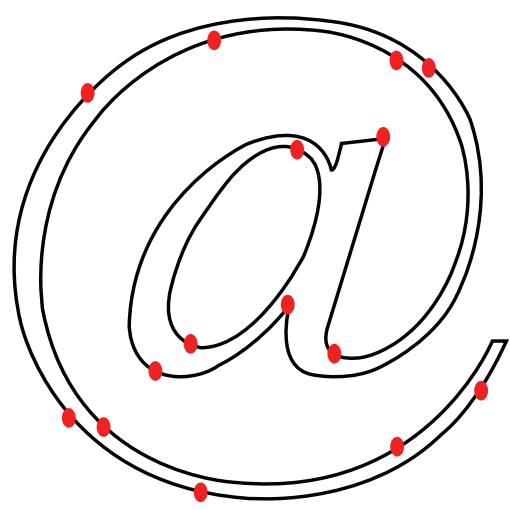

(c)

FIgURE 2: Preprocessing steps: (a) original image, (b) outline of the image, and (c) corner points achieved.

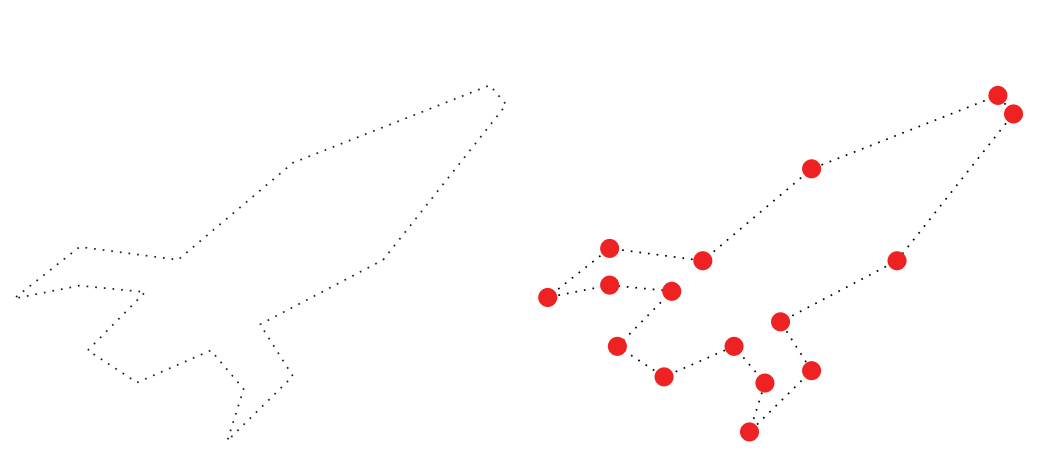

(a) (b)

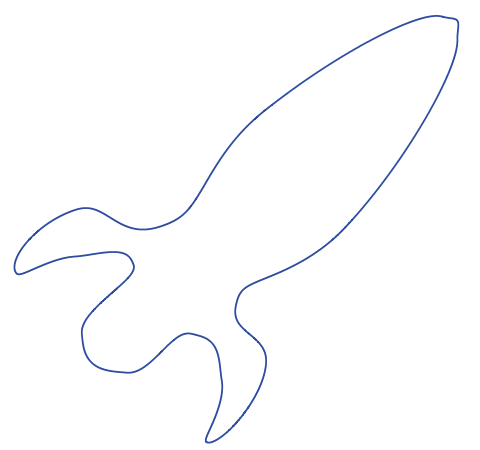

(c)

FIGURE 3: Rational cubic spline interpolant: (a) shape of an image of rocket; (b) depicted data points on the image of rocket; (c) default rational cubic spline curve on the depicted points of the image of rocket.

the method can be seen in Figure 2(b) which is the contour of the bitmap image shown in Figure 2(a).

Corners, in digital images, give important clues for the shape representation and analysis. These are the points that partition the boundary into various segments. The strategy of getting these points is based on the method proposed in [7-9]. The demonstration of the algorithm is made in Figure 2(b). The corner points of the image are shown in Figure 2(c).

\section{Proposed Method}

A piecewise rational cubic spline interpolant $S \in C^{1}\left[t_{i}, t_{i+1}\right]$ with shape parameters $\alpha_{i}, \beta_{i}, \gamma_{i}, \delta_{i}, i=1, \ldots, n$, is defined for $t \in\left[t_{i}, t_{i+1}\right], i=1, \ldots, n$, by

$$
\begin{aligned}
S(t) & =S_{i}(t) \\
& =\frac{U_{i}(1-\theta)^{3}+V_{i} \theta(1-\theta)^{2}+W_{i} \theta^{2}(1-\theta)+X_{i} \theta^{3}}{\alpha_{i}(1-\theta)^{2}+\beta_{i} \theta(1-\theta)^{2}+\gamma_{i} \theta^{2}(1-\theta)+\delta_{i} \theta^{2}},
\end{aligned}
$$

where $t_{1}<t_{2}<, \ldots,<t_{n+1}, h_{i}=t_{i+1}-t_{i}, \theta=\left(t-t_{i}\right) / h_{i}$, and $\alpha_{i}, \beta_{i}, \gamma_{i}, \delta_{i}$ (nonnegative real numbers, but cannot take all zeros at a time) are the shape control parameters for the segment $i$. The rational cubic spline interpolant $(1)$ is $C^{1}$, if it satisfies the following conditions:

$$
\begin{gathered}
S\left(t_{i}\right)=F_{i}, \quad S^{\prime}\left(t_{i}\right)=D_{i}, \\
S\left(t_{i+1}\right)=F_{i+1}, \quad S^{\prime}\left(t_{i+1}\right)=D_{i+1},
\end{gathered}
$$

where $D_{i}, D_{i+1} \in R^{N}$ are the derivative values at the knots $t_{i}$ and $t_{i+1}$. Derivative values $D_{i}$ 's are described in Section 4. Using $C^{1}$ conditions, values of control points $U_{i}, V_{i}, W_{i}$, and $X_{i}$ are as follows:

$$
\begin{gathered}
U_{i}=\alpha_{i} F_{i}, \\
V_{i}=\left(\alpha_{i}+\beta_{i}\right) F_{i}+\alpha_{i} h_{i} D_{i}, \\
W_{i}=\left(\gamma_{i}+\delta_{i}\right) F_{i+1}-\delta_{i} h_{i} D_{i+1}, \\
X_{i}=\delta_{i} F_{i+1} .
\end{gathered}
$$

Remark 1. For $\alpha_{i}=\delta_{i}=1, \beta_{i}=\gamma_{i}=2, i=1, \ldots, n$, the rational cubic spline interpolant (1) reduces to cubic Hermite interpolant. Hence all over this paper, cubic Hermite interpolant is considered as a default case for shape designing. Figure 3(c) demonstrates a default rational cubic spline curve for a shape of image of rocket in Figure 3(a) with depicted data points shown in Figure 3(b). 
3.1. Geometric Properties. The rational cubic spline interpolant (1) satisfies the following geometric properties.

3.1.1. Convex Hull Property. Rational cubic spline interpolant (1) can be written as

$$
\begin{aligned}
S(t)= & S_{i}(t) \\
= & \frac{\alpha_{i} F_{i}(1-\theta)^{3}+\left(\left(\alpha_{i}+\beta_{i}\right) F_{i}+\alpha_{i} h_{i} D_{i}\right) \theta(1-\theta)^{2}}{\alpha_{i}(1-\theta)^{2}+\beta_{i} \theta(1-\theta)^{2}+\gamma_{i} \theta^{2}(1-\theta)+\delta_{i} \theta^{2}} \\
& +\frac{\left(\left(\gamma_{i}+\delta_{i}\right) F_{i+1}-\delta_{i} h_{i} D_{i+1}\right) \theta^{2}(1-\theta)+\delta_{i} F_{i+1} \theta^{3}}{\alpha_{i}(1-\theta)^{2}+\beta_{i} \theta(1-\theta)^{2}+\gamma_{i} \theta^{2}(1-\theta)+\delta_{i} \theta^{2}} .
\end{aligned}
$$

The above equation can be rewritten as

$$
\begin{aligned}
S(t)= & F_{i} R_{0, i}+\left(F_{i}+\frac{\alpha_{i} h_{i} D_{i}}{\left(\alpha_{i}+\beta_{i}\right)}\right) R_{1, i} \\
& +\left(F_{i+1}-\frac{\delta_{i} h_{i} D_{i+1}}{\left(\gamma_{i}+\delta_{i}\right)}\right) R_{2, i}+F_{i+1} R_{3, i}
\end{aligned}
$$

where

$$
\begin{aligned}
R_{0, i} & =\frac{\alpha_{i}(1-\theta)^{3}}{\alpha_{i}(1-\theta)^{2}+\beta_{i} \theta(1-\theta)^{2}+\gamma_{i} \theta^{2}(1-\theta)+\delta_{i} \theta^{2}}, \\
R_{1, i} & =\frac{\left(\alpha_{i}+\beta_{i}\right) \theta(1-\theta)^{2}}{\alpha_{i}(1-\theta)^{2}+\beta_{i} \theta(1-\theta)^{2}+\gamma_{i} \theta^{2}(1-\theta)+\delta_{i} \theta^{2}}, \\
R_{2, i} & =\frac{\left(\gamma_{i}+\delta_{i}\right) \theta^{2}(1-\theta)}{\alpha_{i}(1-\theta)^{2}+\beta_{i} \theta(1-\theta)^{2}+\gamma_{i} \theta^{2}(1-\theta)+\delta_{i} \theta^{2}}, \\
R_{3, i}= & \frac{\delta_{i} \theta^{3}}{\alpha_{i}(1-\theta)^{2}+\beta_{i} \theta(1-\theta)^{2}+\gamma_{i} \theta^{2}(1-\theta)+\delta_{i} \theta^{2}} .
\end{aligned}
$$

One can see that

$$
\begin{aligned}
& R_{j, i} \geq 0, \quad j=0,1,2,3, \\
& R_{0}+R_{1}+R_{2}+R_{3}=1 .
\end{aligned}
$$

Hence the curve segment $S_{i}$ lies in the convex hull of the control points $F_{i}, U_{i}, V_{i}, F_{i+1}$ as shown in Figure 4(a).

3.1.2. Affine Invariance Property. An affine transformation is implemented on the curve by employing it to the control points. Let $x$ be a point in $R^{n}, n=2,3$. An affine transformation denoted by $\varphi$ maps $R^{n}$ into $R^{n}$ and has the form $\varphi(\underline{x})=A \underline{x}+\underline{a} \forall \underline{x} \in R^{n}$ for some $\underline{a} \in R^{n}, n=2,3$, and $A$ is invertible matrix of order $n \times n$. The graphical representation of affine invariance property is shown in Figure 4(b). One can see that image of rocket is not changed in its dimension when it is transformed from one plane to another plane.
Its theoretical proof is as follows. Consider the following equation:

$$
\begin{aligned}
S(t)= & F_{i} R_{0, i}+\left(F_{i}+\frac{\alpha_{i} h_{i} D_{i}}{\left(\alpha_{i}+\beta_{i}\right)}\right) R_{1, i} \\
& +\left(F_{i+1}-\frac{\delta_{i} h_{i} D_{i+1}}{\left(\gamma_{i}+\delta_{i}\right)}\right) R_{2, i}+F_{i+1} R_{3, i} .
\end{aligned}
$$

It can be rewritten as

$$
\begin{aligned}
S(t)= & F_{i} R_{0, i}+\frac{1}{\left(\alpha_{i}+\beta_{i}\right)} V_{i} R_{1, i} \\
& +\frac{1}{\left(\gamma_{i}+\delta_{i}\right)} W_{i} R_{2, i}+F_{i+1} R_{3, i}
\end{aligned}
$$

Now, consider

$\varphi(S(t))$

$$
\begin{gathered}
=\varphi\left(R_{0, i}(\theta) F_{i}+\frac{1}{\left(\alpha_{i}+\beta_{i}\right)} R_{1, i}(\theta) V_{i}\right. \\
\left.\quad+\frac{1}{\left(\gamma_{i}+\delta_{i}\right)} R_{2, i}(\theta) W_{i}+R_{3, i}(\theta) F_{i+1}\right) \\
=A\left(R_{0, i}(\theta) F_{i}+\frac{1}{\left(\alpha_{i}+\beta_{i}\right)} R_{1, i}(\theta) V_{i}\right. \\
\left.\quad+\frac{1}{\left(\gamma_{i}+\delta_{i}\right)} R_{2, i}(\theta) W_{i}+R_{3, i}(\theta) F_{i+1}\right)+\underline{a} \\
=A\left(R_{0, i}(\theta) F_{i}+\frac{1}{\left(\alpha_{i}+\beta_{i}\right)} R_{1, i}(\theta) V_{i}\right.
\end{gathered}
$$

$$
\left.+\frac{1}{\left(\gamma_{i}+\delta_{i}\right)} R_{2, i}(\theta) W_{i}+R_{3, i}(\theta) F_{i+1}\right)
$$

$$
+\sum_{j=0}^{3} R_{j, i}(\theta) \underline{a} \quad\left(\text { This is because } \sum_{j=0}^{3} R_{j, i}(\theta)=1 .\right)
$$$$
=A\left(R_{0, i}(\theta) F_{i}+\frac{1}{\left(\alpha_{i}+\beta_{i}\right)} R_{1, i}(\theta) V_{i}\right.
$$

$$
\begin{aligned}
& \left.+\frac{1}{\left(\gamma_{i}+\delta_{i}\right)} R_{2, i}(\theta) W_{i}+R_{3, i}(\theta) F_{i+1}\right) \\
+ & R_{0, i}(\theta) \underline{a}+R_{1, i}(\theta) \underline{a}+R_{2, i}(\theta) \underline{a} \\
& +R_{3, i}(\theta) \underline{a} \\
= & R_{0, i}(\theta)\left(A F_{i}+\underline{a}\right) \\
+ & R_{1, i}(\theta)\left(A \frac{1}{\left(\alpha_{i}+\beta_{i}\right)} V_{i}+\underline{a}\right)
\end{aligned}
$$




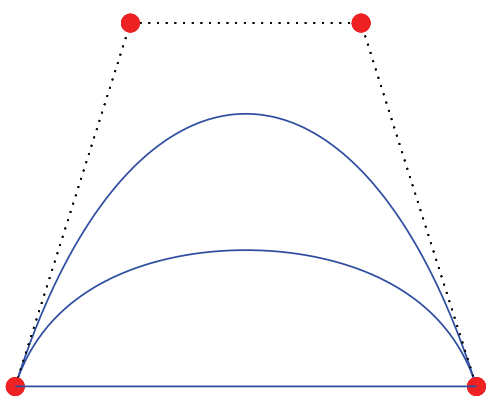

(a)

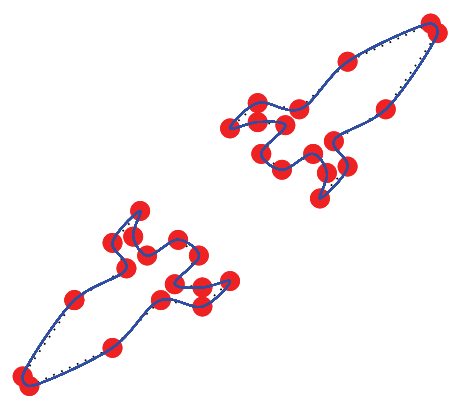

(b)

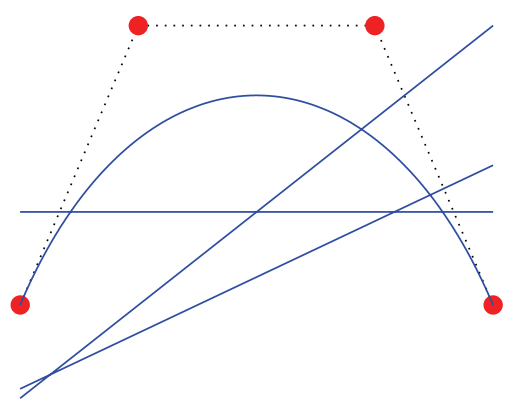

(c)

FiguRE 4: Rational cubic spline interpolant: (a) convex hull property; (b) affine invariance property; and (c) variation diminishing property.

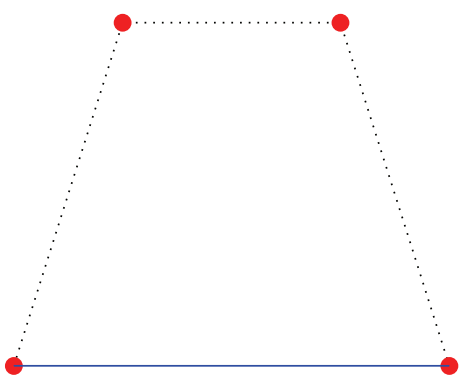

(a)

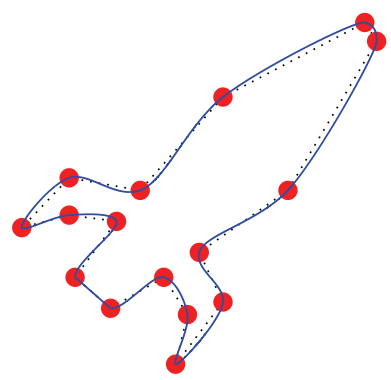

(b)

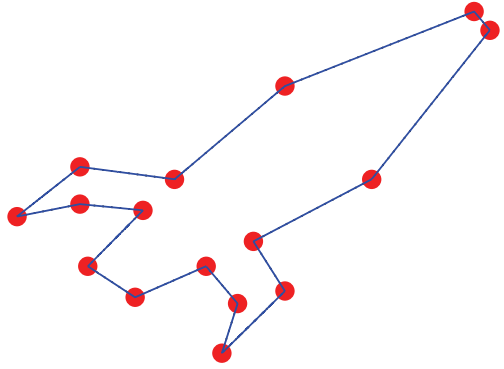

(c)

FIGURE 5: Rational cubic spline interpolant: (a) linear interpolant, that is, $\alpha_{i}, \delta_{i} \rightarrow 0$, and (b) interval tension property, that is, $\alpha_{k}, \delta_{k} \rightarrow 0$, $k=10$. (c) Global tension property, that is, $\alpha_{i}, \delta_{i} \rightarrow 0, \forall i$.

$$
\begin{aligned}
& +R_{2, i}(\theta)\left(A \frac{1}{\left(\gamma_{i}+\delta_{i}\right)} W_{i}+\underline{a}\right) \\
& +R_{3, i}(\theta)\left(A F_{i+1}+\underline{a}\right) \\
& =R_{0, i}(\theta) \varphi\left(F_{i}\right)+R_{1, i}(\theta) \varphi\left(\frac{1}{\left(\alpha_{i}+\beta_{i}\right)} V_{i}\right) \\
& +R_{2, i}(\theta) \varphi\left(\frac{1}{\left(\gamma_{i}+\delta_{i}\right)} W_{i}\right) \\
& +R_{3, i}(\theta) \varphi\left(F_{i+1}\right) .
\end{aligned}
$$

Hence

$$
\begin{aligned}
\varphi(S(t))= & R_{0, i}(\theta) F_{i}^{*}+R_{1, i}(\theta) \frac{1}{\left(\alpha_{i}+\beta_{i}\right)} V_{i}^{*} \\
& +R_{2, i}(\theta) \frac{1}{\left(\gamma_{i}+\delta_{i}\right)} W_{i}^{*}+R_{3, i}(\theta) F_{i+1}^{*},
\end{aligned}
$$

where

$$
\begin{aligned}
F_{i}^{*} & =\varphi\left(F_{i}\right), \\
\frac{1}{\left(\alpha_{i}+\beta_{i}\right)} V_{i}^{*} & =\varphi\left(\frac{1}{\left(\alpha_{i}+\beta_{i}\right)} V_{i}\right),
\end{aligned}
$$

$$
\begin{aligned}
\frac{1}{\left(\gamma_{i}+\delta_{i}\right)} W_{i}^{*} & =\varphi\left(\frac{1}{\left(\gamma_{i}+\delta_{i}\right)} W_{i}\right), \\
F_{i+1}^{*} & =\varphi\left(F_{i+1}\right) .
\end{aligned}
$$

3.1.3. Variation Diminishing Property. The curve segment $S_{i}$ crosses any plane of dimension $N-1$ not more often than it crosses the control polygon joining $F_{i}, U_{i}, V_{i}, F_{i+1}$ as shown in Figure 4(c).

3.1.4. Linear Interpolant. For $\alpha_{i}, \delta_{i} \rightarrow 0$ and $\beta_{i},=\gamma_{i}=1$ the rational cubic spline interpolant (1) converges to the linear interpolant $L_{i}(t)$ where $L_{i}(t)=F_{i}(1-\theta)+\theta F_{i+1}$. This is practically shown in Figure 5(a).

3.1.5. Global Tension Property. Let $L \in C^{0}\left[t_{1}, t_{n+1}\right]$ denote the piecewise linear interpolant defined for $t \in\left[t_{i}, t_{i+1}\right]$ by $L(t)=$ $L_{i}(t)$. Then, for $\alpha_{i}, \delta_{i} \rightarrow 0, \forall i$,

$$
L(t) \equiv L_{i}(t)=F_{i}(1-\theta)+\theta F_{i+1}, \quad i=1,2, \ldots, n-1 .
$$

This is practically shown in Figure 5(b).

3.1.6. Interval Tension Property. Let $D_{i}$ 's be bounded and consider an interval $\left[t_{k}, t_{k+1}\right]$ for a fixed $k \in 1, \ldots, n$. Then on $\left[t_{k}, t_{k+1}\right]$ with fixed $\alpha_{i}, \delta_{i}, i \neq k$ when $\alpha_{k}, \delta_{k} \rightarrow 0,\left\|S_{k}-L_{k}\right\|=$ 0 as shown in Figure 5(c).

It is observed in Figure 6 that the shape of the curve can be controlled by assigning the different values to the free 


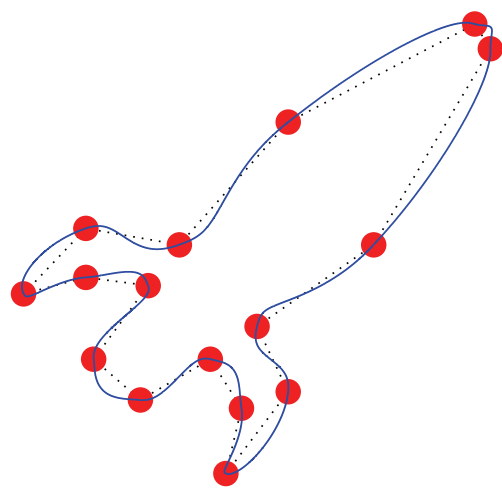

(a)

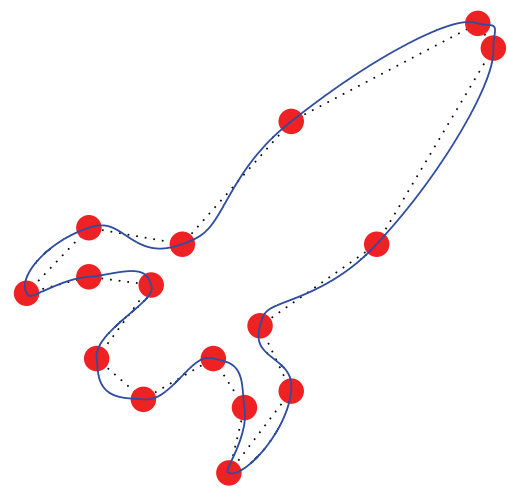

(d)

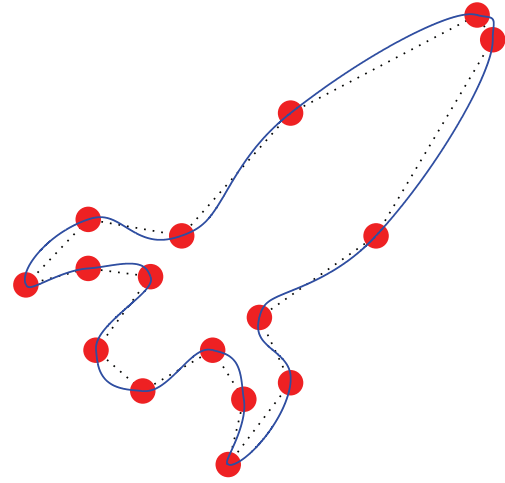

(b)

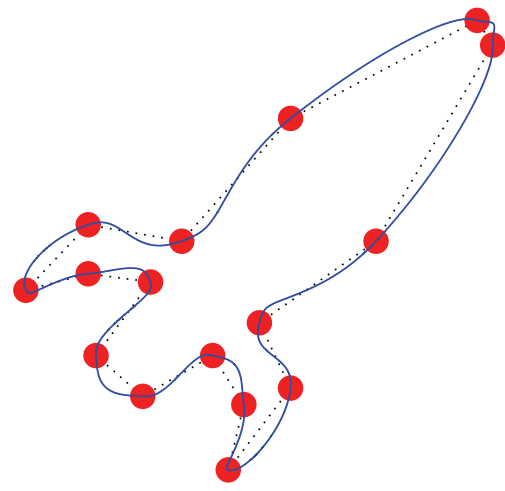

(e)

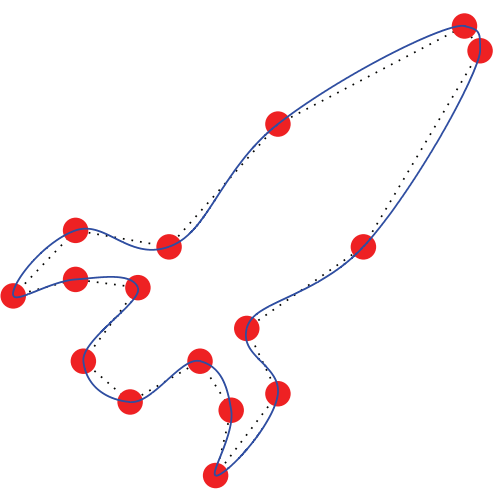

(c)

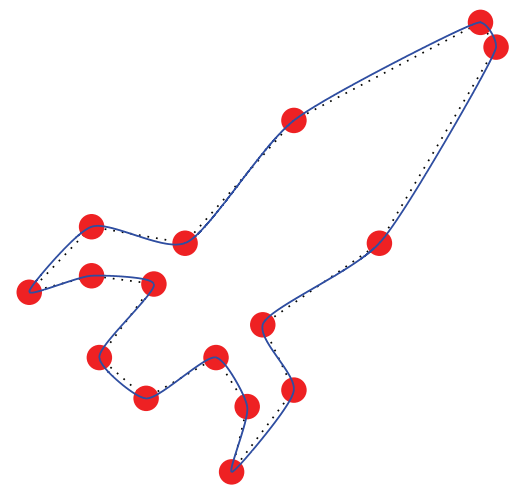

(f)

FIGURE 6: Effect of the parameters on the shape of the curve: (a) $\alpha_{i}=100, \beta_{i}=2, \gamma_{i}=2, \delta_{i}=100$; (b) $\alpha_{i}=10, \beta_{i}=2, \gamma_{i}=2, \delta_{i}=10$; (c) $\alpha_{i}=2, \beta_{i}=2, \gamma_{i}=2, \delta_{i}=2$. Effect of the parameters on the shape of the curve: (d) $\alpha_{i}=1, \beta_{i}=0.001, \gamma_{i}=0.001, \delta_{i}=1 ;(\mathrm{e}) \alpha_{i}=10, \beta_{i}=0.01$, $\gamma_{i}=0.01, \delta_{i}=10$; (f) $\alpha_{i}=2, \beta_{i}=0.9, \gamma_{i}=0.9, \delta_{i}=2$.

parameters $\alpha_{i}, \beta_{i}, \gamma_{i}, \delta_{i}$ in the description of rational cubic spline interpolant (1). In Figures 6(a), 6(b), and 6(c), it is observed that the shape of the curve is loose and far from the control polygon for $\alpha_{i}=100, \delta_{i}=100$ and is becoming tightened by decreasing the value of parameters $\alpha_{i}, \delta_{i}$. In Figures 6(d), 6(e), and 6(f), it is observed that the shape of the curve is loose and far from the control polygon for $\beta_{i}=0.001$, $\gamma_{i}=0.001$ and is becoming tightened by increasing the value of parameters $\beta_{i}, \gamma_{i}$.

The curve segment varies between the parameter values $t_{i}$ and $t_{i+1}$ depending on the shape control parameters $\alpha_{i}, \beta_{i}, \gamma_{i}, \delta_{i}$ and the derivatives at the control points. Effect of the derivatives (at the end points) on the shape of the curve is given in Figure 7. Figures 7(a), 7(b), and 7(c) show simple, twisted, and loop curves, respectively.

Consider a window of the car (rectangular polygon) in Figure 8. In Figure 8(a), blue, red, and green curves are obtained using rational cubic spline interpolant (1) with $\alpha_{i}=$ $1, \beta_{i}=2, \gamma_{i}=2, \delta_{i}=1, \alpha_{i}=10, \beta_{i}=2, \gamma_{i}=2, \delta_{i}=1$, and $\alpha_{i}=1, \beta_{i}=2, \gamma_{i}=2, \delta_{i}=10$, respectively. It can be observed from the figure that, by changing the value of $\alpha_{i}$, it effects the curve near the start of the control point and by changing the value of $\delta_{i}$ it effects the curve near the end of the control point. That is, biased behavior is also part of the scheme for controlling curves from the two ends of the pieces. Thus, it shows that rational spline interpolant having four parameters gives more control to the user to modify or refine the figure up to a satisfactory level.

Consider again the window of the car (rectangular polygon), in Figures 8(b) and 8(c), for clear and better understanding of global and interval tension properties for rational cubic spline interpolant. Figure $8(\mathrm{~b})$ shows global tension property for the values of parameters $\alpha_{i}=\delta_{i}=$ $100,10,5,1,0.5,0.1,0.001$ with $\beta_{i}=\gamma_{i}=2$ as black, pink, magenta, green, red, yellow, and dark blue, respectively. Figure 8(c) shows interval tension property for values of parameters $\alpha_{2}=\delta_{2}=100,10,5,1,0.5,0.1,0.001$ with $\beta_{i}=$ $\gamma_{i}=2$ as black, pink, magenta, green, red, yellow, and dark blue colors, respectively.

Figure 9 is showing image of a car for designing, with a variety of options for shape parameters in their formations.

\section{Choice of Derivatives}

The proposed rational parametric splines have derivative values in their descriptions. If these derivative values are not given, then these derivative values are usually estimated through various numerical techniques like arithmetic mean technique, geometric mean technique, or harmonic mean technique to implement splines. In this paper, arithmetic mean technique is used to estimate the derivative values and the following subsections elaborate these notions. 


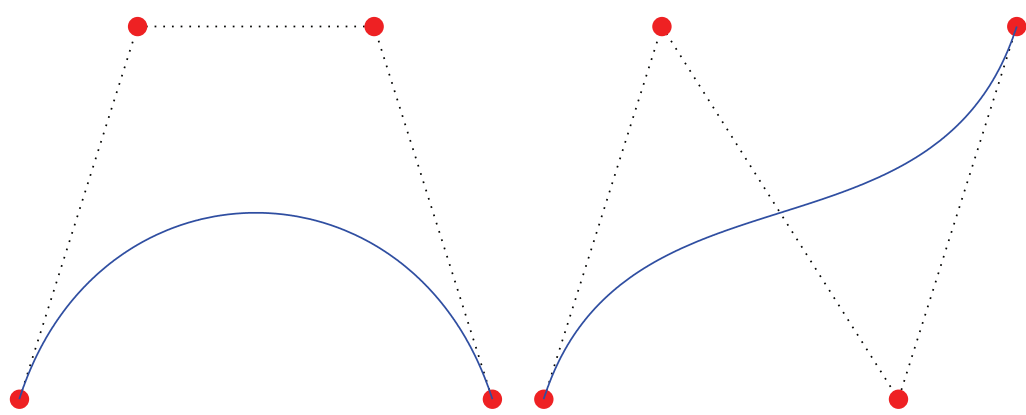

(a) (b)

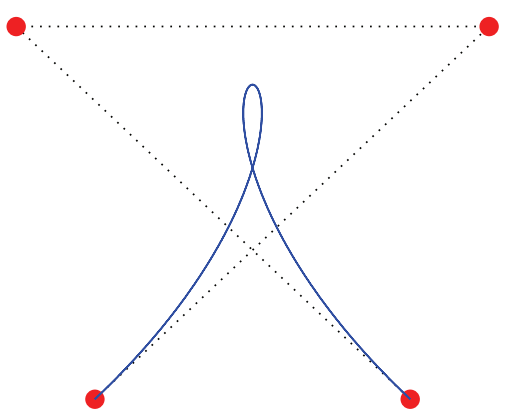

(c)

FIGURE 7: Effect of the derivatives (at the end points) on the shape of the curve: (a) simple curve; (b) twisted curve; (c) loop curve.

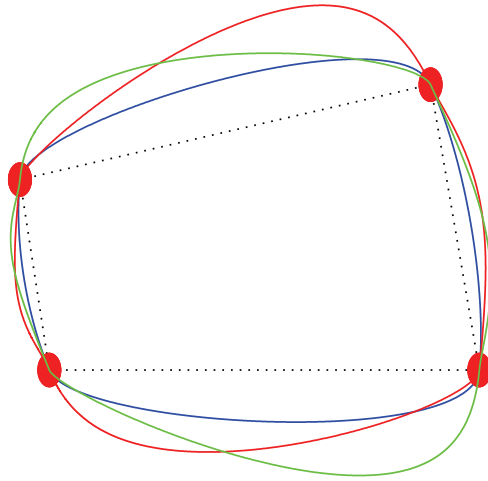

(a)

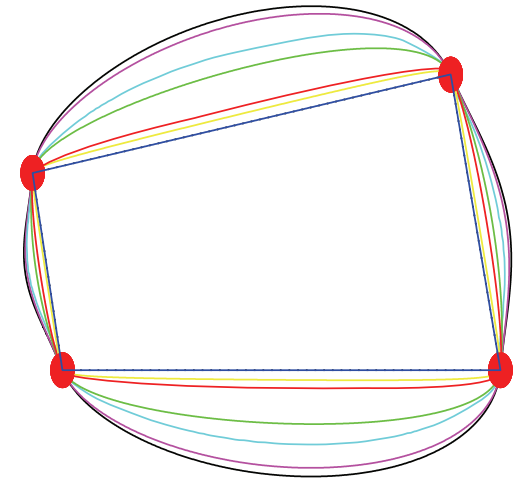

(b)

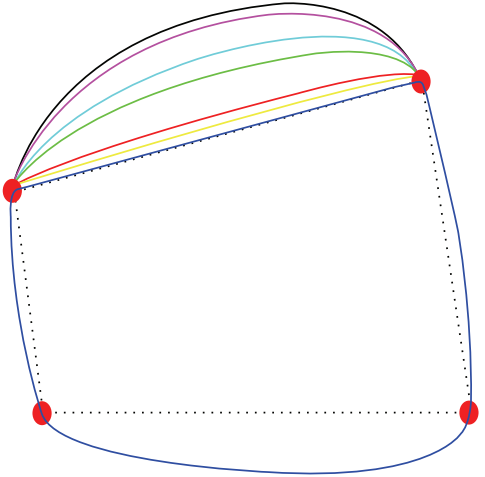

(c)

FIGURE 8: Rational spline interpolants: (a) effect of parameters; (b) global interval tension property; (c) local interval tension property.

4.1. Cyclic Closed Curves. A $n$ segment curve with $n+1$ control points is said to be closed curve with $C^{1}$ continuity if the control points $F_{1}$ and $F_{n+1}$ and the derivative values $D_{1}$ and $D_{n+1}$ are equal to each other; that is, $F_{1}=F_{n+1}, D_{1}=D_{n+1}$. Here, all $D_{i}$ 's are estimated with Arithmetic Means method [13].

4.2. Anticyclic Closed Curves. A $n$ segment curve with $n+1$ control points is said to be closed curve with $C^{1}$ continuity if the control points $F_{1}$ and $F_{n+1}$ are equal to each other and the derivative values $D_{1}$ and $D_{n+1}$ are also equal in magnitude but opposite in direction; that is, $F_{1}=F_{n+1}, D_{1}=-D_{n+1}$. Here, all $D_{i}$ 's are estimated with Arithmetic Means method [13].

\section{Surface Engineering and Designing}

The proposed work is one of the best candidates to be extended for the designing of surfaces for CAD/CAM/CAE and engineering images/symbols. The extension of the curve scheme to tensor product surface representations is immediately apparent. This surface presents a spline surface with shape parameters. However, this representation exhibits a problem common to all tensor product descriptions in that the shape control parameters affect a complete row or column of the tensor product array.

We propose a tensor product like approach [19] but actually it is not a tensor product. Instead of step functions, the tension weights are introduced as $C^{2}$ continuous splines in the description of the tensor product. It causes producing local control, in the construction of surfaces, in an independent way. The details of the proposed method are out of the scope of this paper and will be discussed somewhere else.

\section{Reasons to Have New Spline Scheme}

The existing spline schemes, in the current literature, have limited features as far as shape designing is concerned. In its individual capacity, each of the existing spline methods has either one type of shape control (point tension or interval tension) or two types of shape controls (point and interval tensions). The proposed curve design scheme is distinct from the existing spline methods in various ways. It represents a parametric rational cubic spline with the most general description. It has three types of shape controls (point, interval, and biased tensions). This general description is quite helpful to produce well-controlled shape effects as compared to any existing spline methods [5, 10, 19-27].

The proposed method provides a large variety of very interesting shape controls like point, interval, and biased tensions (at both ends). Moreover, as special cases, it also recovers various splines including the cubic splines [21], the rational cubic spline with tension [26], the rational cubic spline in [5], another rational cubic spline in [19], and another rational cubic spline in [10]. On top of it, the proposed 

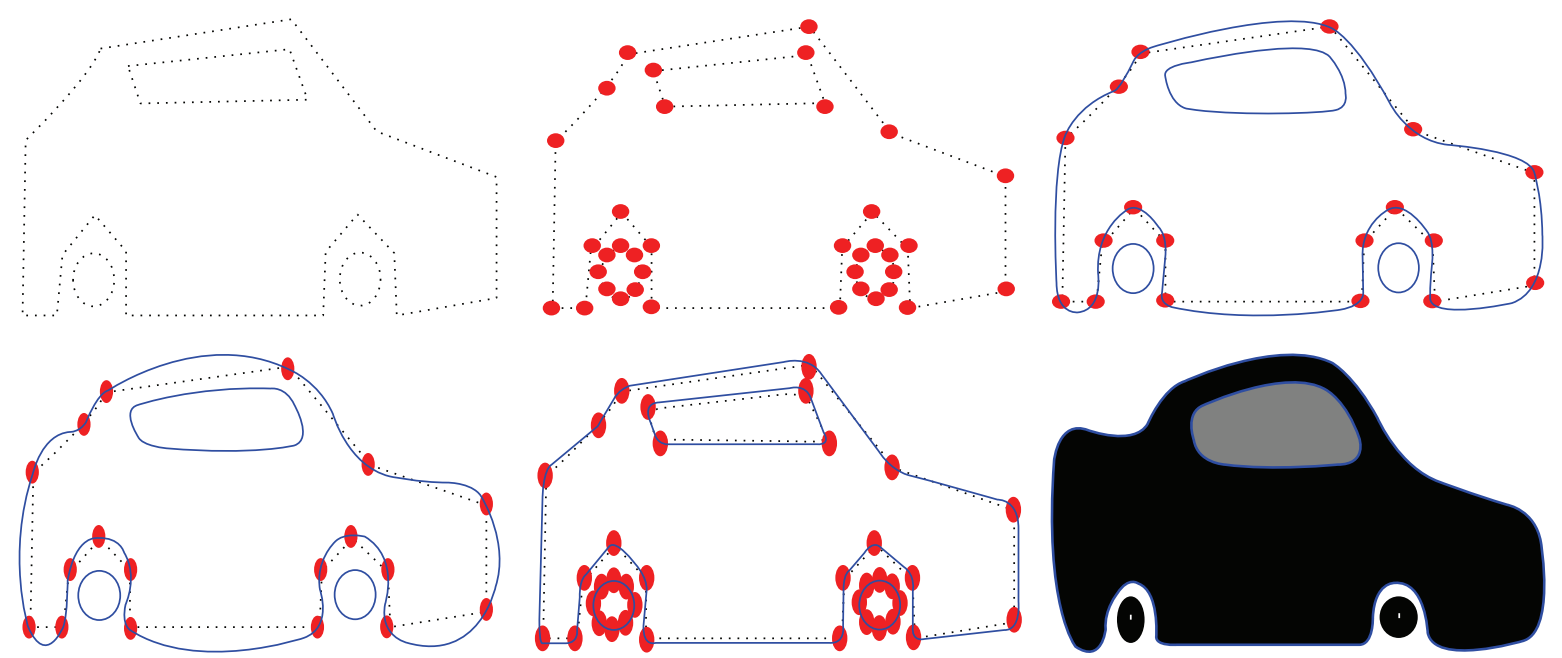

FIGURE 9: Rational spline interpolants for the design of a car.

method serves as a stronger alternative to its cubic spline counterparts. For example, it works as $C^{1}$ alternative to the $G C^{1}$ or $C^{1}$ cubic spline methods like $v$-splines [23] because of point tension, beta splines [25] because of point and interval tensions (and partly for biased tension in one direction), gamma splines [24] because of interval tension, and weighted splines [22] because of weak interval tension. It is worth mentioning that the point, interval, and biased tensions, in the proposed scheme, are well controlled and to the point as compared to their counterparts of $v$-splines, gamma splines, weighted splines, and beta splines. The mathematical analysis of such facts is under consideration of the authors at this stage, and it is not explained here due to fear of too much length of the paper. These mathematical descriptions will be addressed at some other stage in another paper.

\section{Conclusion}

This paper suggests and contributes towards shape designing and reengineering of engineering of images using spline interpolants. A piecewise rational cubic spline interpolant with free parameters is developed for this purpose. Practical examples are taken into account to demonstrate the proposed scheme. Favorable geometrical properties of the interpolant have also been discussed in detail together with pictorial demonstrations. The method provides a variety of shape control to show that user and designer can get a control on the image locally as well as globally. Using local control properties, one can obtain control on the image without disturbing the whole figure. It represents a parametric rational cubic spline with the most general description. It has three types of shape controls (point, interval, and biased tensions). This general description is quite helpful to produce well-controlled shape effects as compared to any existing spline methods. The proposed method also serves as an alternate to various splines including $v$-splines, gamma splines, weighted splines, and beta splines.

As the work is in progress, many aspects of the proposed system need to be developed further in several manners.
Firstly, a comprehensive set of datasets need to be collected. The datasets should include all significant engineering images involving $2 \mathrm{D}$ and $3 \mathrm{D}$ aspects. Secondly, the algorithm needs to be improved to find out automatic choices of shape parameters to provide the desired outlines. All the parameters that are being used in the devised algorithm need to be adjusted for suitability and appropriateness. This should be such that the algorithm performs the best with extensive datasets.

\section{Conflict of Interests}

The authors declare that there is no conflict of interests regarding the publication of this paper.

\section{Acknowledgment}

The authors are indebted to the anonymous referees whose valuable comments made this paper more useful.

\section{References}

[1] How to Design an Engineering Library, http://www.ehow.com/ info_8175983_major-stages-engineering-design.html.

[2] Engineering Images, https://www.google.com.pk/search?q=engineering+images\&espv $=2 \&$ es_sm $=122 \&$ source $=\operatorname{lnms\& tbm}=$ isch\&sa $=$ X\&ei $=u$ UyFVPStOYm7UeWUg9gP\&ved $=0$ CAgQ AUoAQ\&biw $=1365 \& b i h=902 \& \mathrm{dpr}=0.75$.

[3] M. S. Ameer and A. H. F. Hossam, "Meta-designing parameterized Arabic fonts for AlQalam," TUGboat Proceeding of the Annual Meeting, vol. 29, no. 3, pp. 435-443, 2008.

[4] F. Arrebola and F. Sandoval, "Corner detection and curve segmentation by multiresolution chain-code linking," Pattern Recognition, vol. 38, no. 10, pp. 1596-1614, 2005.

[5] Z. Habib, M. Sakai, and M. Sarfraz, "Interactive shape control with rational cubic splines," Computer-Aided Design and Applications, vol. 1, no. 1-4, pp. 709-717, 2004. 
[6] A. K. Murtaza, "An automated algorithm for approximation of temporal video data using linear Bezier fitting," The International Journal of Multimedia \& Its Applications, vol. 2, no. 2, pp. 81-94, 2010.

[7] M. Sarfraz, M. Z. Hussain, M. Irshad, and A. Khalid, "Approximating boundary of bitmap characters using genetic algorithm," in Proceedings of the 7th International Conference on Computer Graphics, Imaging and Visualization (CGIV '10), vol. 21, pp. 8691, August 2010.

[8] M. Sarfraz, M. Irshad, and M. Z. Hussain, "Vectorization of image outlines using rational spline and genetic algorithm," International Proceedings of Computer Science and Information Technology, vol. 50, pp. 16-20, 2012.

[9] M. Sarfraz, M. R. Asim, and A. Masood, "Capturing outlines using cubic bezier curves," in Proceedings of the International Conference on Information and Communication Technologies: From Theory to Applications (ICTTA '04), pp. 539-540, IEEE, April 2004.

[10] M. Sarfraz and M. Balah, "A curve design method with shape control," in Computational Science and Its Applications-ICCSA, V. Kumar, M. L. Gavrilova, C. J. K. Tan, and P. L' Ecuyer, Eds., vol. 2669 of Lecture Notes in Computer Science, pp. 670-679, Springer, Berlin, Germany, 2003.

[11] M. Sarfraz and M. A. Khan, "An automatic outline fitting algorithm for arabic characters," in Computational Science and Its Applications-ICCSA 2003, vol. 2669 of Lecture Notes in Computer Science, pp. 589-598, Springer, Berlin, Germany, 2003.

[12] M. Sarfraz, "Optimal curve fitting to digital data," Journal of WSGC, vol. 11, no. 1, pp. 394-401, 2003.

[13] M. Sarfraz, M. Z. Hussain, and M. Ishaq, "Modeling of objects using conic splines," Journal of Software Engineering and Applications, vol. 6, no. 3, pp. 67-72, 2013.

[14] M. Sarfraz, "Two approaches for vectorizing image outlines," International Journal of Machine Learning and Computing, vol. 2, no. 3, pp. 301-307, 2012.

[15] X. Yang and W. Yang, "Cone spline approximation via fat conic spline fitting," Computer Aided Design, vol. 38, no. 6, pp. 703712, 2006.

[16] L. Zhu, C. Zhu, J. Pei, X. Li, and W. Wang, "Prediction of threedimensional milling forces based on finite element," Advances in Materials Science and Engineering, vol. 2014, Article ID 918906, 7 pages, 2014.

[17] E. Mohseni, E. Zalnezhad, A. A. D. Sarhan, and A. R. Bushroa, "A study on surface modification of Al7075-T6 alloy against fretting fatigue phenomenon," Advances in Materials Science and Engineering, vol. 2014, Article ID 474723, 17 pages, 2014.

[18] S.-C. Li, Z.-Z. Chen, J. Zheng, and D.-F. Wang, "Smooth particle hydrodynamics simulation of micro-cup-extrusion using a graphit-ic coating," Advances in Materials Science and Engineering, vol. 2014, Article ID 863028, 6 pages, 2014.

[19] M. Sarfraz, "Curves and surfaces for computer aided design using $C^{2}$ rational cubic splines," Engineering with Computers, vol. 11, no. 2, pp. 94-102, 1995.

[20] M. Sarfraz, M. Z. Hussain, and M. Hussain, "Shape-preserving curve interpolation," International Journal of Computer Mathematics, vol. 89, no. 1, pp. 35-53, 2012.

[21] G. E. Farin, Curves and Surfaces for Computer Aided Geometric Design, Academic Press, New York, NY, USA, 1997.

[22] T. A. Foley, "Local control of interval tension using weighted splines," Computer Aided Geometric Design, vol. 3, no. 4, pp. 281294, 1986.
[23] G. M. Nielson, "A locally controllable spline with tension for interactive curve design," Computer Aided Geometric Design, vol. 1, no. 3, pp. 199-205, 1984.

[24] W. Boehm, "Curvature continuous curves and surfaces," Computer Aided Geometric Design, vol. 2, no. 4, pp. 313-323, 1985.

[25] W. Boehm, "Rational geometric splines," Computer Aided Geometric Design, vol. 4, no. 1-2, pp. 67-77, 1987.

[26] J. A. Gregory and M. Sarfraz, "A rational cubic spline with tension," Computer Aided Geometric Design, vol. 7, no. 1-4, pp. 1-13, 1990.

[27] B. A. Barsky, The beta-spline: a local representation based on shape parameters and fundamental geometric measure [Ph.D. thesis], University of Utah, 1981. 

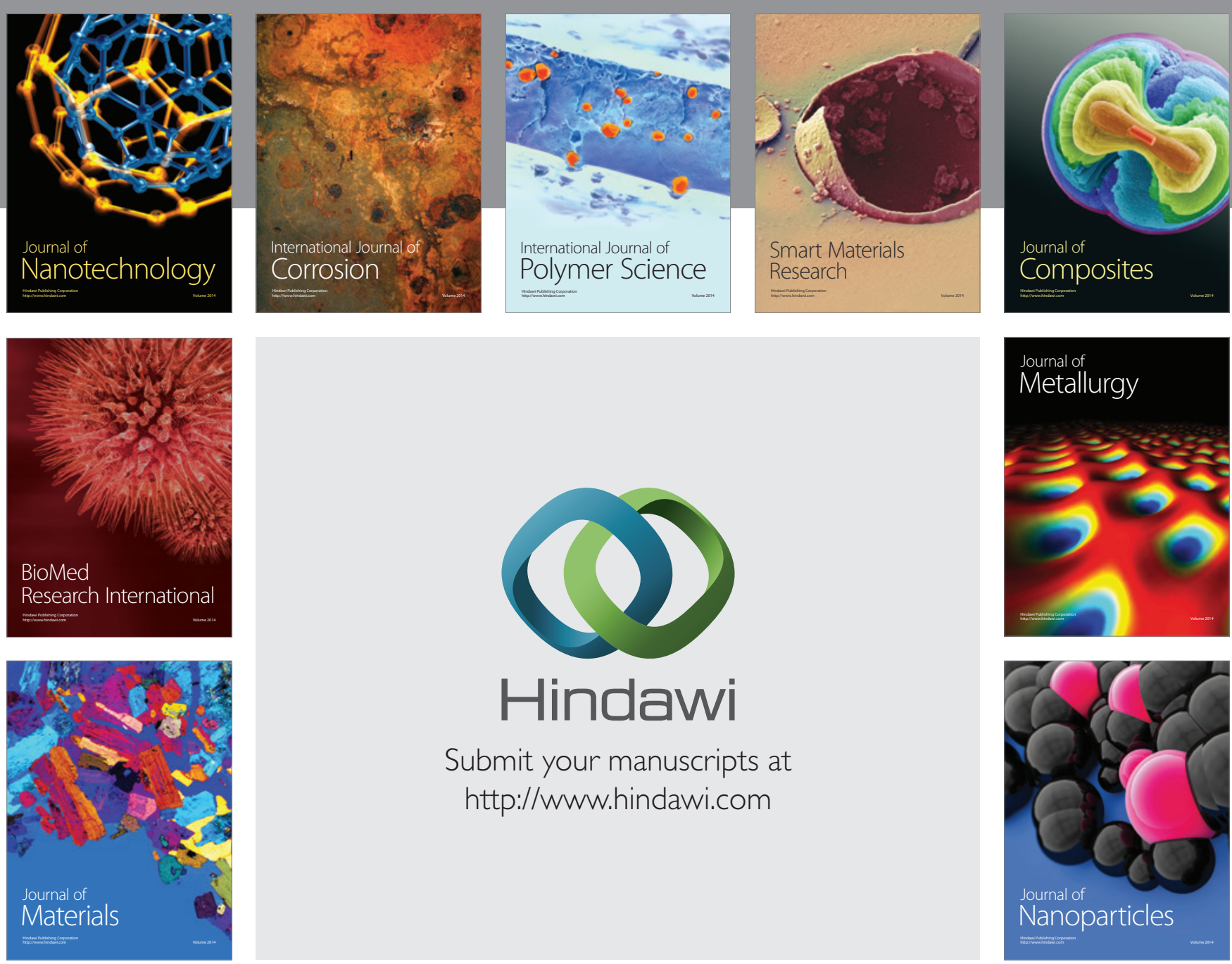

Submit your manuscripts at http://www.hindawi.com
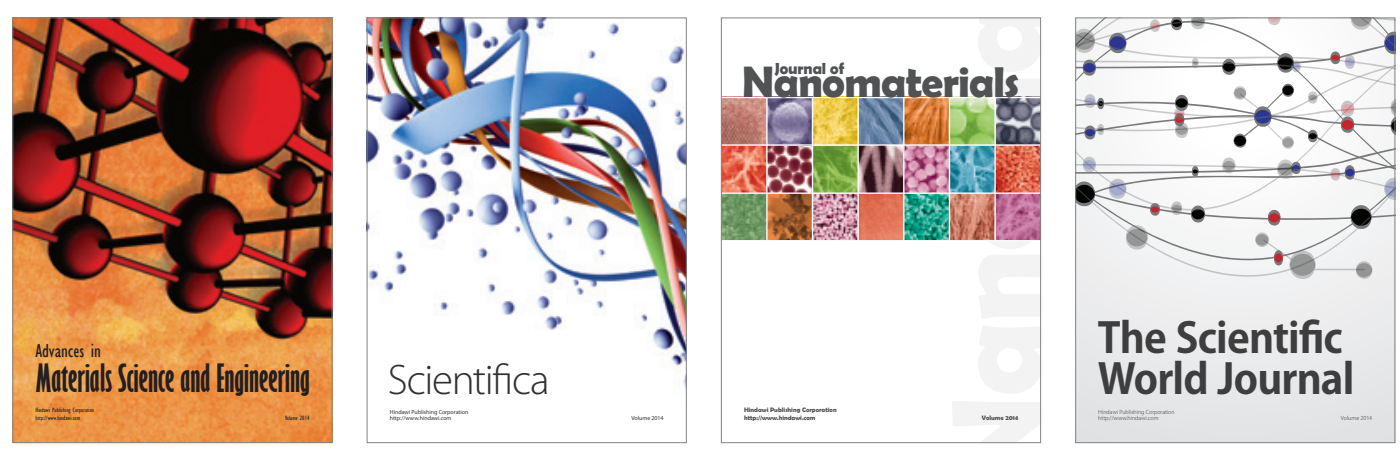

\section{The Scientific World Journal}
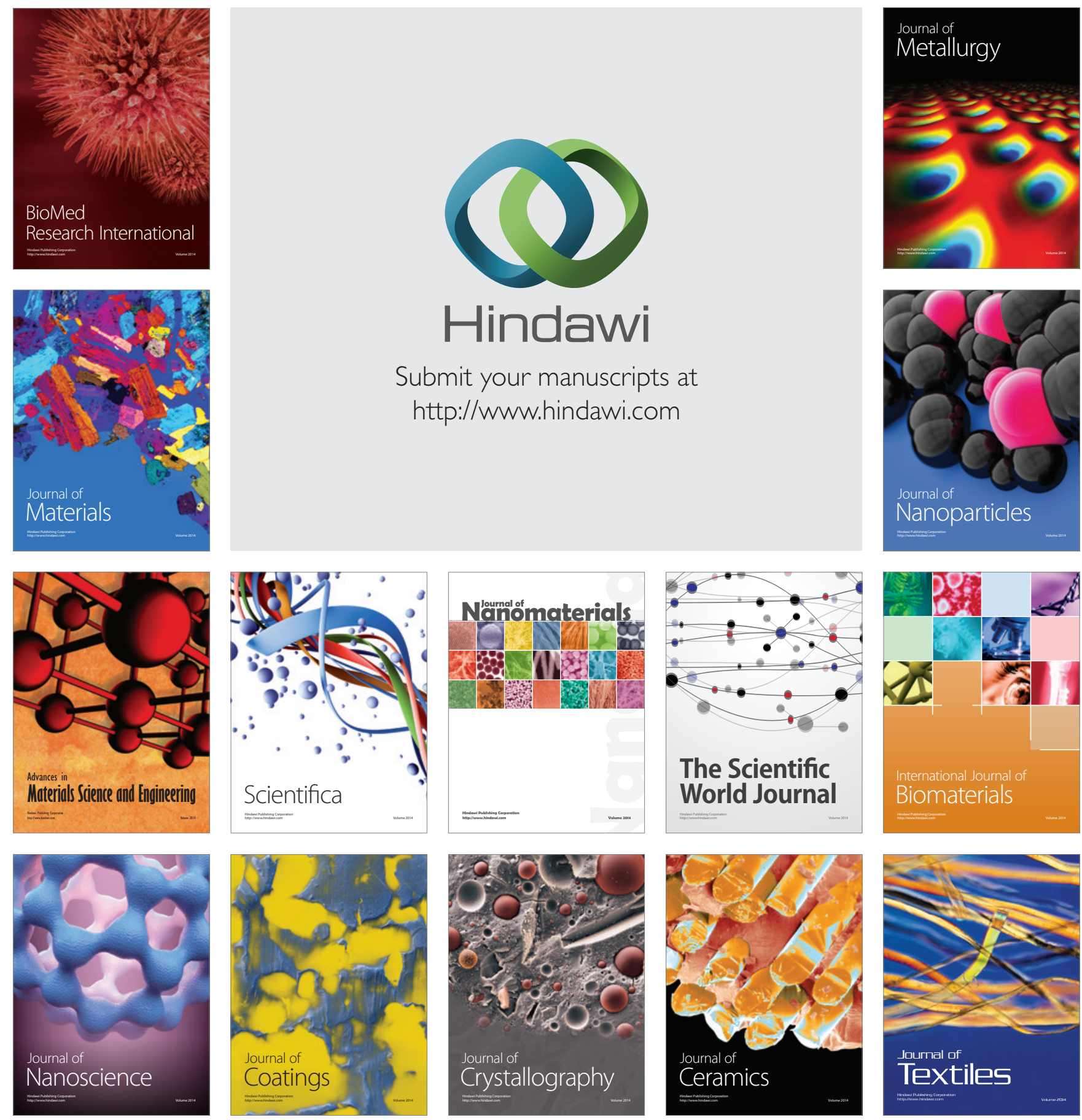